\title{
Room Temperature Molecular Single-Electron Transistor
}

\author{
E. S. Soldatov ${ }^{1}$, V. V. Khanin ${ }^{1}$, A. S. Trifonov ${ }^{1}$, S. P. Gubin ${ }^{2}$, V. V. Kolesov ${ }^{3}$, D. E. \\ Presnov $^{1}$, S. A. Iakovenko ${ }^{1}$, G. B. Khomutov ${ }^{1}$, and A. N. Korotkov ${ }^{4}$. \\ ${ }^{1}$ Department of Physics, Moscow State University, Moscow 119899, Russia, \\ ${ }^{2}$ Institute of General and Inorganic Chemistry, Moscow 11790\%, Russia, \\ ${ }^{3}$ Institute of Radioengineering and Electronics, Moscow 103907, Russia, \\ and ${ }^{4}$ Nuclear Physics Institute, Moscow State University, Moscow 119899, Russia
}

(February 24, 2018)

\begin{abstract}
A room temperature single-electron transistor based on the single cluster molecule has been demonstrated for the first time. Scanning tunneling microscope tip was used to study the transport via single carboran cluster molecule 1,7-( $\left(\mathrm{CH}_{3}\right)_{2}-1,2-\mathrm{C}_{2} \mathrm{~B}_{10} \mathrm{H}_{9} \mathrm{Tl}\left(\mathrm{OCOCF}_{3}\right)_{2}$ incorporated into the Langmuir-Blodgett monolayer of the stearic acid. DC $I-V$ curves at $300 \mathrm{~K}$ showed well pronounced Coulomb staircase, and the current could be controlled by a lithographically fabricated gold gate electrode.
\end{abstract}

73.40.Gk, 61.16.Ch, 36.40.Cg

Typeset using REVTEX 
A considerable interest was attracted to the effects of correlated single-electron tunneling [1] during the last decade. The most of experiments with lithographically fabricated structures were done at temperatures below $1 \mathrm{~K}$. To increase the operating temperature $T$ it is necessary to reduce the characteristic size $d$ of the structure in order to decrease the typical capacitance $C$. Operation at $T=300 \mathrm{~K}$ requires $C \lesssim 10^{-18} \mathrm{~F}$ corresponding to $d \lesssim 3 \mathrm{~nm}$, quite a difficult task. Several different fabrication techniques were studied to obtain singleelectron effects at relatively high temperatures (for a recent review see, e.g., Ref. [2]). Let us mention, for example, room temperature memory effects [3], a single-electron transistor with visible charging effects up to $300 \mathrm{~K}$ 四 using silicon-based structures, a gated system of metal grains [5] operating at $77 \mathrm{~K}$, and room-temperature single-electron transistor fabricated by nanooxidation process [6].

Another technique in which the small capacitances are easily obtained is based on scanning tunneling microscopy (STM). The simplest single-electron circuit consisting of two tunnel junctions in series can be implemented using the STM tip, small conducting particle, and the substrate. The single-electron charging survives up to room temperature for sufficiently small metal particles [7,8], and it can be even stronger when the tunneling via single molecules is studied [9 [12]. The drawback of this technique was the absence of the gate electrode to control the transport. In the present letter we report the first demonstration of a room temperature molecular single-electron transistor (the schematic is shown in Fig. 1) with a metallic gate controlling the electron tunneling from the STM tip to the substrate via single carboran cluster molecule (see also Ref. [13]).

The molecules with the "core" consisting of the cluster of metal atoms and surrounded by ligands are being extensively studied by both chemical and physical methods during last few decades (see, e.g., Ref. [14] and references therein). The important property of these molecules is their ability to accept as well as to release several electrons without the structural change. The similar quasimetallic property can be found in some cluster molecules with nonmetallic core [14]. In our experiment the Tl-derivative 1,7- $\left(\mathrm{CH}_{3}\right)_{2}-1,2-$ $\mathrm{C}_{2} \mathrm{~B}_{10} \mathrm{H}_{9} \mathrm{Tl}\left(\mathrm{OCOCF}_{3}\right)_{2}$ of carboran (which has the nonmetallic core) was used (for brevity 
we call this cluster molecule simply as carboran).

Langmuir-Blodgett (LB) monolayers of stearic acid with incorporated cluster molecules were deposited on the pyrolytic graphite (HOPG) substrate with pre-formed gate electrode. This electrode was fabricated by the conventional electron lithography and presented a system of thin $(50 \mathrm{~nm})$ and narrow $(400 \mathrm{~nm})$ gold strips separated by 400-nm gaps from each other (Fig. 2). All strips were electrically connected and separated from the substrate by a $50 \mathrm{~nm}$ thick insulator $\left(\mathrm{Al}_{2} \mathrm{O}_{3}\right)$ - see Fig. 1 .

The LB monolayer was deposited using a computerized version on the conventional teflon trough by the technique described in more detail in Refs. [11] and [15] (see also Ref. [16]). The small volume (about $0.1 \mathrm{ml}$ ) of the mixture (1:36) of the carboran clusters and the stearic acid dissolved in tetrahydrofuran (total molecular concentration $0.001 \mathrm{M}$ ) was put on the surface of MilliQ-purified water. After the complete evaporation of tetrahydrofuran the remaining monolayer was compressed at a speed about $5 \AA^{2} /$ molecule/min. The LB monolayer was transferred from the trough onto the substrate with gate electrode by Schaefer's method [17.

Electron transport through the film was studied at room temperature by NanoScope STM having an atomic resolution. First the films were imaged at the typical tip bias voltage of $0.1 \mathrm{~V}$ and the tunneling current of $0.5 \mathrm{nA}$. The images were stable and reproducible. The carboran clusters were seen as elevated oval shape objects with the longer size about $2 \mathrm{~nm}$ and the shorter size crudely twice less. The average distance between clusters was about 20 $\mathrm{nm}$. The size and the shape were not strictly reproducible, that can be explained, e.g., by the different orientations of the cluster molecules in the monolayer.

After finding a cluster molecule, the STM tip was positioned above the cluster and a series of the transport measurements with disconnected STM feedback loop was carried out (the feedback loop with voltage of $0.1 \mathrm{~V}$ and current of $0.5 \mathrm{nA}$ was used between measurements to restore the vertical position of the tip). Actually the measurements were carried in 49 closely located points in the plane, so that the points directly above the cluster as well as the points aside the cluster were studied (similar technique was used in Ref. [1]). The typical 
dc $I$ - $V$ curve in the case of tunneling via the cluster molecule is shown in Fig. 3. It has a clear staircase shape (with voltage period about $130 \mathrm{mV}$ ) while there are no stairs if the STM tip is far from the cluster. Figure 3 also shows the result of the differential conductance measurement using lock-in technique. Notice that the voltage position of the conductance oscillations corresponds well to the staircase structure, however, the measured conductance is somewhat larger than what it could be expected from the numerical derivative of the $I-V$ curve. The difference can be explained by the slight deviation of the tip position between the measurements (the vertical drift is about $0.6 \AA /$ sec and in-plane drift is about $0.2 \AA /$ sec; the duration of one $I$ - $V$ curve measurement is about $10 \mathrm{msec}$ while one conductance curve measurement takes about 3 seconds). Another reason is the fact that the curves in Fig. 3 actually show the characteristics averaged over 4 independent sweeps (the individual curves were typically too noisy).

The curve 1 in Figure 4 shows a dependence of the tunneling current $I$ on the gate voltage $V_{g}$ for the case when the STM tip was positioned above the cluster located about 60 $\mathrm{nm}$ from the gate electrode. The curve is clearly periodic with the period about $0.8 \mathrm{~V}$. In contrast, $I-V_{g}$ curves do not show such an effect when the STM tip is positioned above the flat area of surface without clusters (curve 2). Assuming that each period of the oscillations of the curve 1 corresponds to one additional electron, we can estimate the charge sensitivity of our molecular single-electron transistor as $10^{-3} e / \mathrm{Hz}^{1 / 2}$ (the measurement system had a bandwidth of $16 \mathrm{kHz}$ ). We suppose that mechanical vibrations give the main contribution to the total system noise ( 150 pA peak-to-peak in Fig. 田). The modulation amplitude of the $I-V_{g}$ curve depends on the dc bias voltage $V$. Qualitatively we have found that the amplitude is maximal for $V$ corresponding to the points between the steps of $I$ - $V$ curve while the gate control is almost negligible when $V$ corresponds to a flat part of the curve. We should note that the clearly periodic control curves for the tunneling via cluster were observed quite rare (curve 1 in Fig. 1 presents the best result), that complicated their quantitative analysis.

The experimental results qualitatively agree with the theory of the single-electron transis- 
tor [1], however, several issues are not clear yet. The single electron tunneling in a molecular system should necessarily be affected [2.18] by the discreteness of the energy spectrum. In this case the electric capacitance becomes a not well-defined quantity, and the Coulomb energy converts into the energy of ionization and electron affinity. However, at the present time there is no independent information in the literature about the energy spectrum of the studied system. On the other hand, the simple "orthodox" theory [1] which assumes the continuous energy spectrum of the electrodes is proven to work sufficiently well even for nanometer-size systems.

So, as a first approximation it is natural to try the explanation of the experimental $I-V$ curve shape using the "orthodox" theory. Then taking the staircase period of $\Delta V \simeq 130$ $\mathrm{mV}$ from Fig. [3 we can calculate the capacitance of the junction with larger resistance as $C=e / \Delta V \simeq 1.2 \times 10^{-18} \mathrm{~F}$. This value is too large to be explained as the capacitance of the core of the carboran cluster which is $0.7 \mathrm{~nm}$ in diameter. STM image of the cluster molecule is also considerably larger (about $2 \mathrm{~nm}$ ). It is reasonable to assume that the effective size (in the sense of electric charging energy) is larger that the core size [19]. The sphere of the diameter $d=2 \mathrm{~nm}$ has capacitance $C \simeq \varepsilon \times 1.1 \times 10^{-19} \mathrm{~F}$. Hence, the experimental capacitance of $1.2 \times 10^{-18} \mathrm{~F}$ can be reasonably well explained if we assume the effective dielectric constant $\varepsilon \sim 5$ (related to the substrate, stearic acid, and adsorbate) and take into account the capacitance increase when geometry is closer to the plane capacitor.

Figure 5 shows the $I-V$ curves calculated using the "orthodox" theory (curves 1 and 2 ) to fit the experimental result (curve 3). One can see that the agreement between the theory and the experiment is quite good qualitatively but not perfect quantitatively. The calculations show that to have so well pronounced Coulomb staircase the temperature should be more than 1.5 times smaller than it was experimentally. Almost horizontal steps obtained in the experiment can be explained in the "orthodox" theory assuming that the tunnel junction which has much higher resistance, has also considerably larger capacitance. Though such an assumption does not seem to be quite natural, it is the usual explanation of the almost flat steps in single-electron experiments using STM (see, e.g., Ref. [20]). The horizontal steps 
could be also explained if we assume the discrete energy spectrum of the central electrode of transistor [18], that is quite natural for the molecular system.

The gate capacitance $C_{g}$ calculated from the period of the control curve (curve 1 in Fig. (1) is about $2 \times 10^{-19} \mathrm{~F}$. The ratio $C_{g} / C \sim 0.15$ is relatively large although the distance between the cluster and the gate electrode $(\sim 60 \mathrm{~nm})$ is much larger than the typical distance between the STM tip and the cluster molecule. The possible explanation can be based on the fact that the graphite substrate is quite far from being a perfect conductor, and this considerably reduces the screening of the gate voltage by the bias electrodes. The theoretical fitting of the modulation amplitude of the control curve 1 shown in Fig. 国 (using the capacitance calculated from Fig. (3) gives the discrepancy of about 1.5 times in the temperature similar to the discrepancy in the $I$ - $V$ curve fitting. However, Fig. $\mathbb{Q}$ shows the largest current swing obtained, and the typical control curves do not go beyond the limit explainable by the "orthodox" theory.

In conclusion, we have demonstrated the controllable single-electron system based on a single cluster molecule. The clear Coulomb staircase and the transistor action were obtained at room temperature. The experimental results are in a good qualitative agreement with the "orthodox" theory of the single-electron transistor, however, there are several quantitative discrepancies. Further studies of this subject are certainly necessary.

The work was supported in part by the Russian program on the prospective technologies for nanoelectronics (Grant No. 5-029/26/1-3), the Russian program on the physics of nanostructures (Grant No. 1-091/4) and the Russian Foundation for Basic Research (Grant No. 96-03-33766a). 


\section{REFERENCES}

[1] D. V. Averin and K. K. Likharev, in Mesoscopic Phenomena in Solids, edited by B. L. Altshuler, P. A. Lee, and R. A. Webb (Elsevier, Amsterdam, 1991), p. 173.

[2] A. N. Korotkov, to be published in Molecular Electronics, edited by J. Jortner and M. A. Ratner (Blackwell, Oxford).

[3] K. Yano, T. Ishii, T. Hashimoto, T. Kobayashi, F. Murai, and K. Seki, IEEE Trans. on Electron. Dev. 41, 1628 (1994).

[4] Y. Takahashi, M. Nagase, H. Namatsu, K. Kurihara, K. Iwdate, Y. Nakajima, S. Horiguchi, K. Mirase, and M. Tabe, Electron. Lett. 31, No. 2, 136 (1995).

[5] W. Chen and H. Ahmed, J. Vac. Sci. Technol. B 13, 2883 (1995).

[6] K. Matsumoto, M. Ishii, K. Segawa, Y. Oka, B. J. Vartanian, and J. S. Harris, Appl. Phys. Lett. 68, 34 (1996).

[7] C. Schönenberger, H. van Houten, and H. C. Donkersloot, Europhys. Lett. 20, 249 (1992).

[8] M. Dorogi, J. Gomes, R. Osifchin, R. P. Andres, R. Reifenberger, Phys. Rev. B 52, 9071 (1995).

[9] H. Nejoh, Nature 353, 640 (1991).

[10] C. M. Fischer, M. Burghard, S. Roth, and K. v. Klitzing, Europhys. Lett. 28, 129 (1994).

[11] A. A. Zubilov, S. P. Gubin, A. N. Korotkov, A. G. Nikolaev, E. S. Soldatov, V. V. Khanin, G. B. Khomutov, and S. A. Yakovenko, Tech. Phys. Lett. 20, 195 (1994).

[12] J. G. A. Dubois, J. W. Gerritsen, S. E. Shafranjuk, E. J. G. Boon, G. Schmid, and H. van Kempen, Europhys. Lett. 33, 279 (1996). 
[13] E. S. Soldatov, V. V. Khanin, A. S. Trifonov, S. P. Gubin, V. V. Kolesov, D. E. Presnov, S. A. Iakovenko, and G. B. Khomutov, Pis'ma Zh. Eksp. Teor. Fiz. 64, No. 8, 510 (1996) [JETP Lett.].

[14] U. Kreibig and M. Vollmer, Optical properties of metal clusters (Springer, Berlin, 1995).

[15] S. A. Iakovenko, A. S. Trifonov, E. S. Soldatov, V. V. Khanin, S.P.Gubin, and G. B. Khomutov, Thin Solid Films, 284-285, 873 (1996).

[16] S. P. Gubin, E. S. Soldatov, A. S. Trifonov, and V. V. Khanin, Inorg. Mat. 32, 1265 (1996). S. A. Iakovenko, S. P. Gubin, E. S. Soldatov, A. S. Trifonov, V. V. Khanin, G. B. Khomutov, Inorg. Mat. 32, 1272 (1996).

[17] G.L. Gaines, Insoluble monolayers at liquid-gas interfaces (Interscience Publ., N. Y, 1966).

[18] D. V. Averin and A. N. Korotkov, Sov. Phys. JETP 70, 937 (1990).

[19] We should note that in our earlier study of the similar system [11] (without gate electrode) we did not observe the pronounced Coulomb staircase, but observed considerably larger $(\sim 500 \mathrm{mV})$ Coulomb blockade. It is not excluded that additional fabrication step has changed the chemical environment of the cluster molecules.

[20] R. Wilkins, E. Ben-Jacob, and R. C. Jaklevic, Phys. Rev. Lett. 63, 801 (1989). 


\section{FIGURES}

FIG. 1. Schematic of the single-electron transistor based on the single cluster molecule. 1 HOPG substrate, 2 - insulating layer $\left(\mathrm{Al}_{2} \mathrm{O}_{3}\right), 3$ - gold gate electrode.

FIG. 2. SEM image of the gold gate electrode fabricated before the monolayer deposition.

FIG. 3. The typical $I$ - $V$ curve and the differential conductance (as functions of the bias voltage $V)$ of the molecular single-electron transistor.

FIG. 4. Curve 1 - the dependence of the current through the molecular single-electron transistor on the gate voltage (dc bias point is shown by arrow in Fig. 3). Curve 2 - similar dependence when STM tip is positioned above the stearic acid (without cluster). The bias voltage for both curves is $0.2 \mathrm{~V}$.

FIG. 5. The $I-V$ curves calculated using the "orthodox" theory (curves 1 and 2 ) to fit the experimental result (curve 3). Curves are shifted vertically for clarity. 


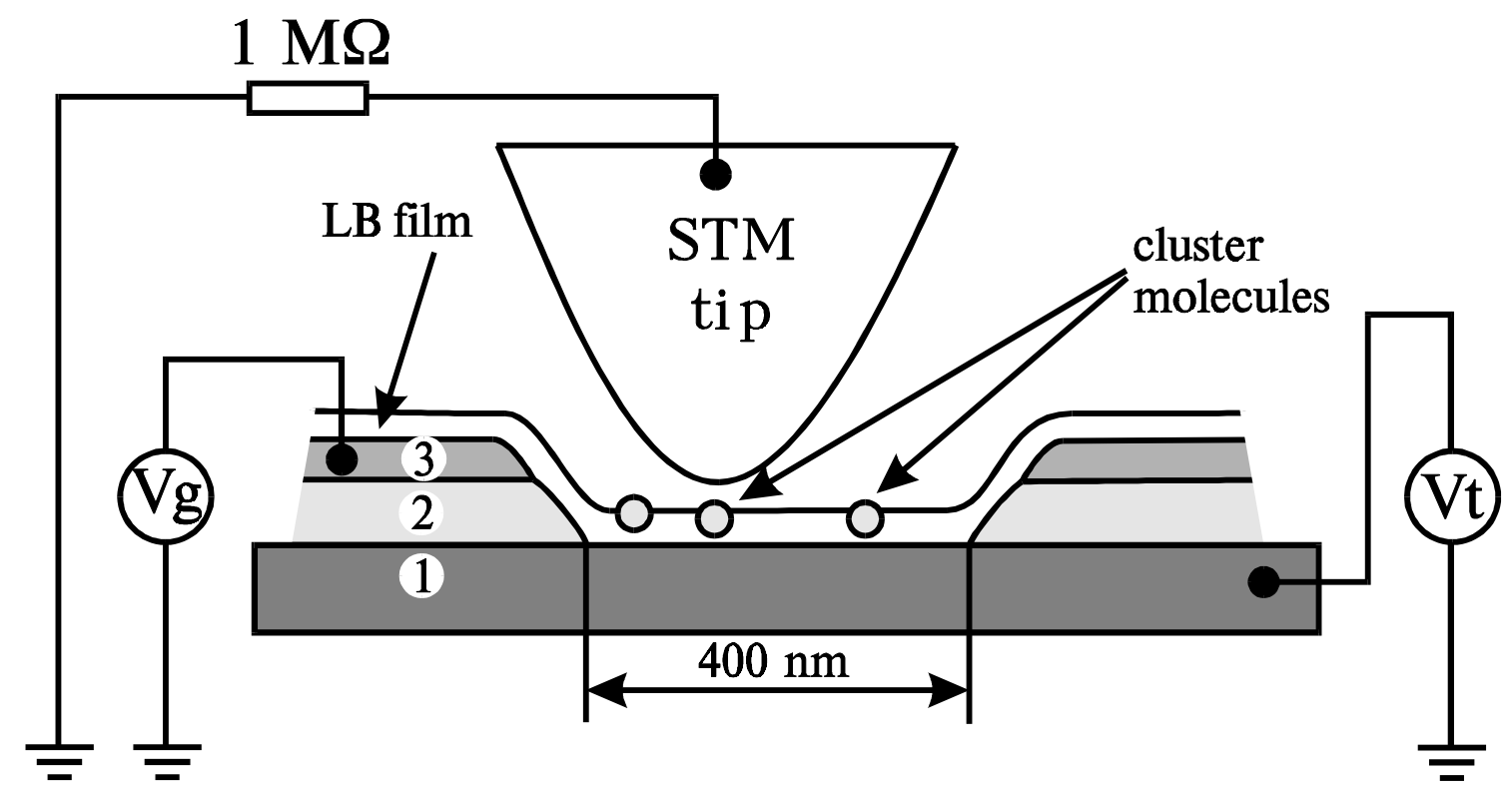




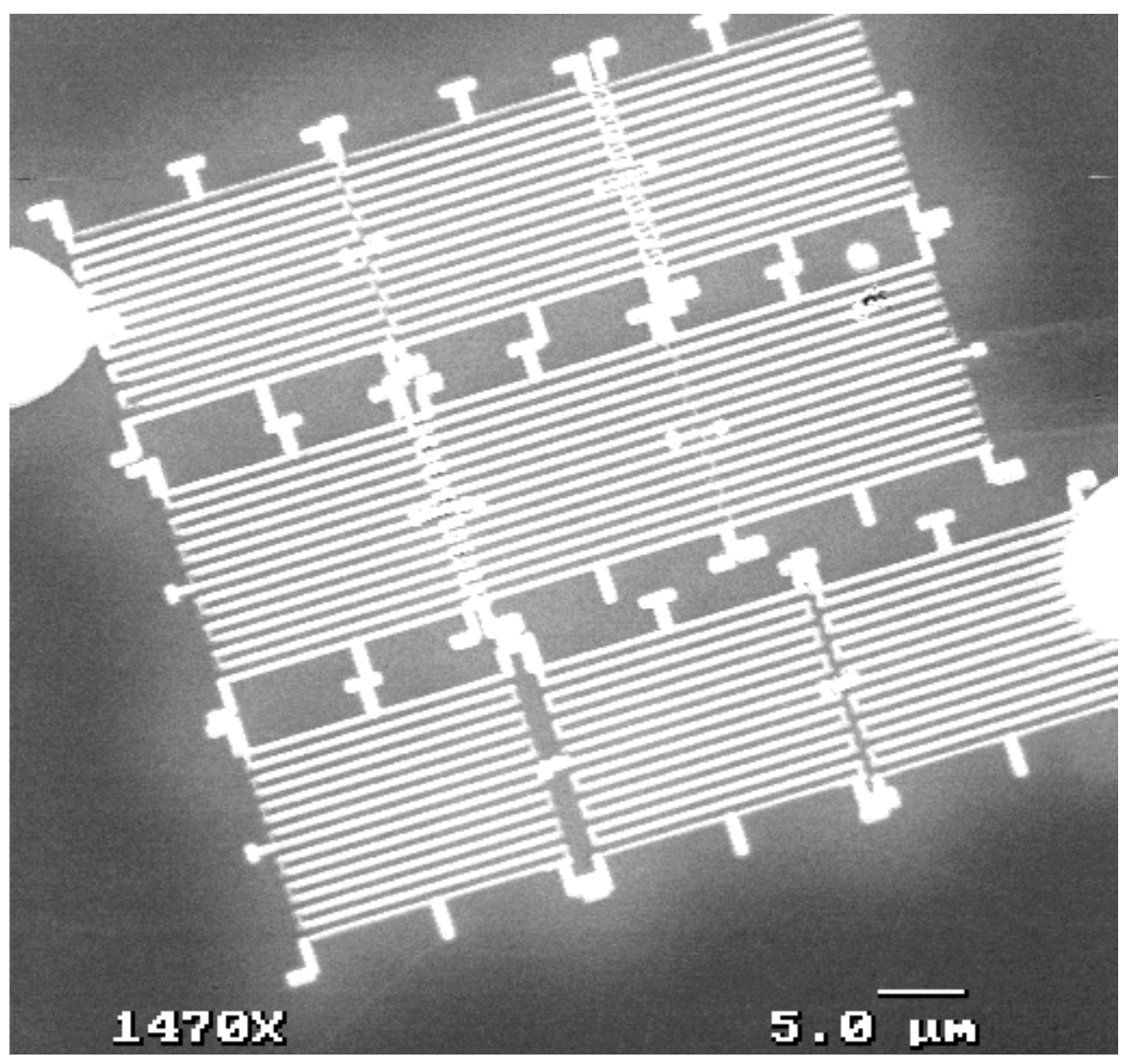




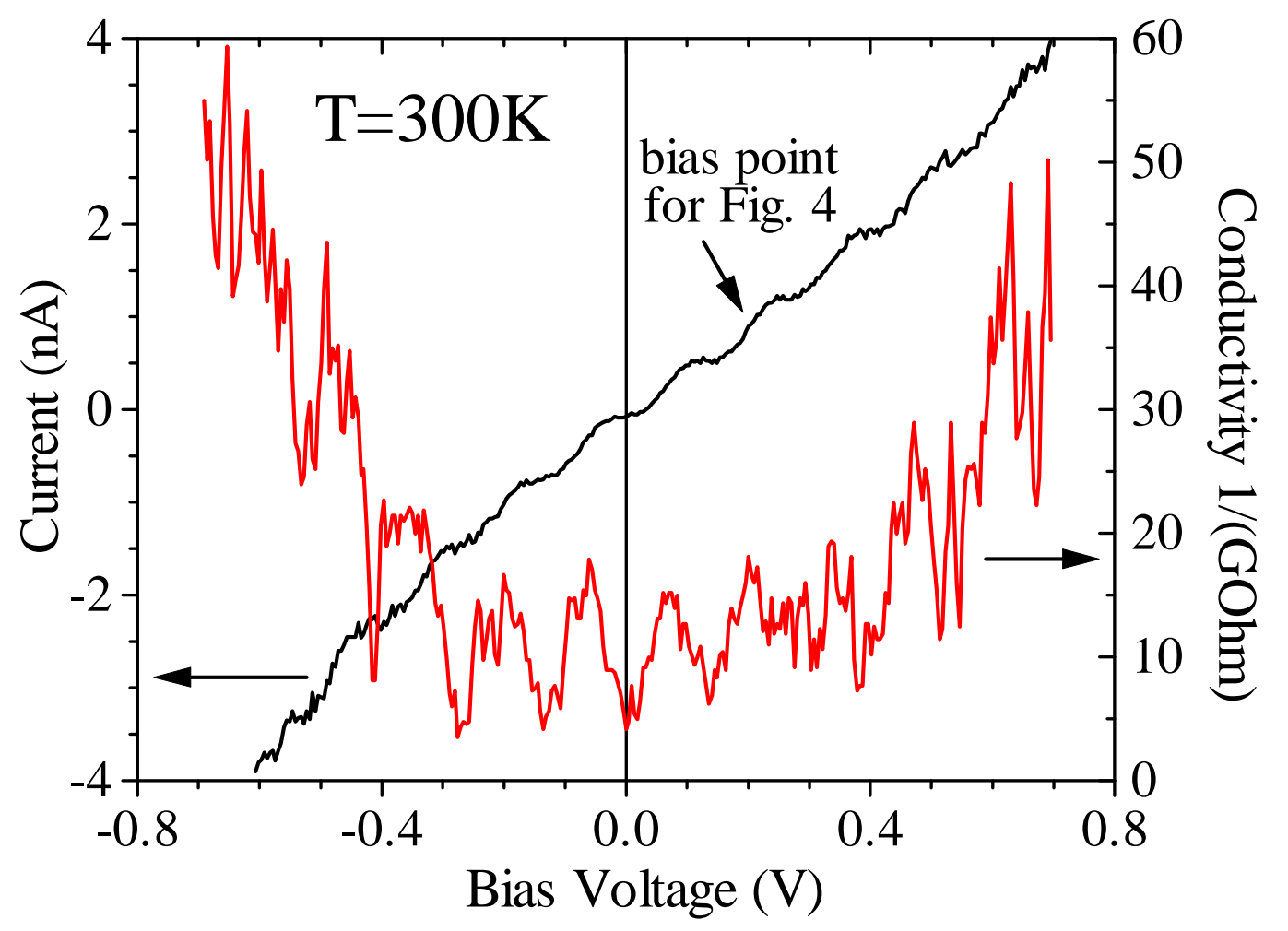




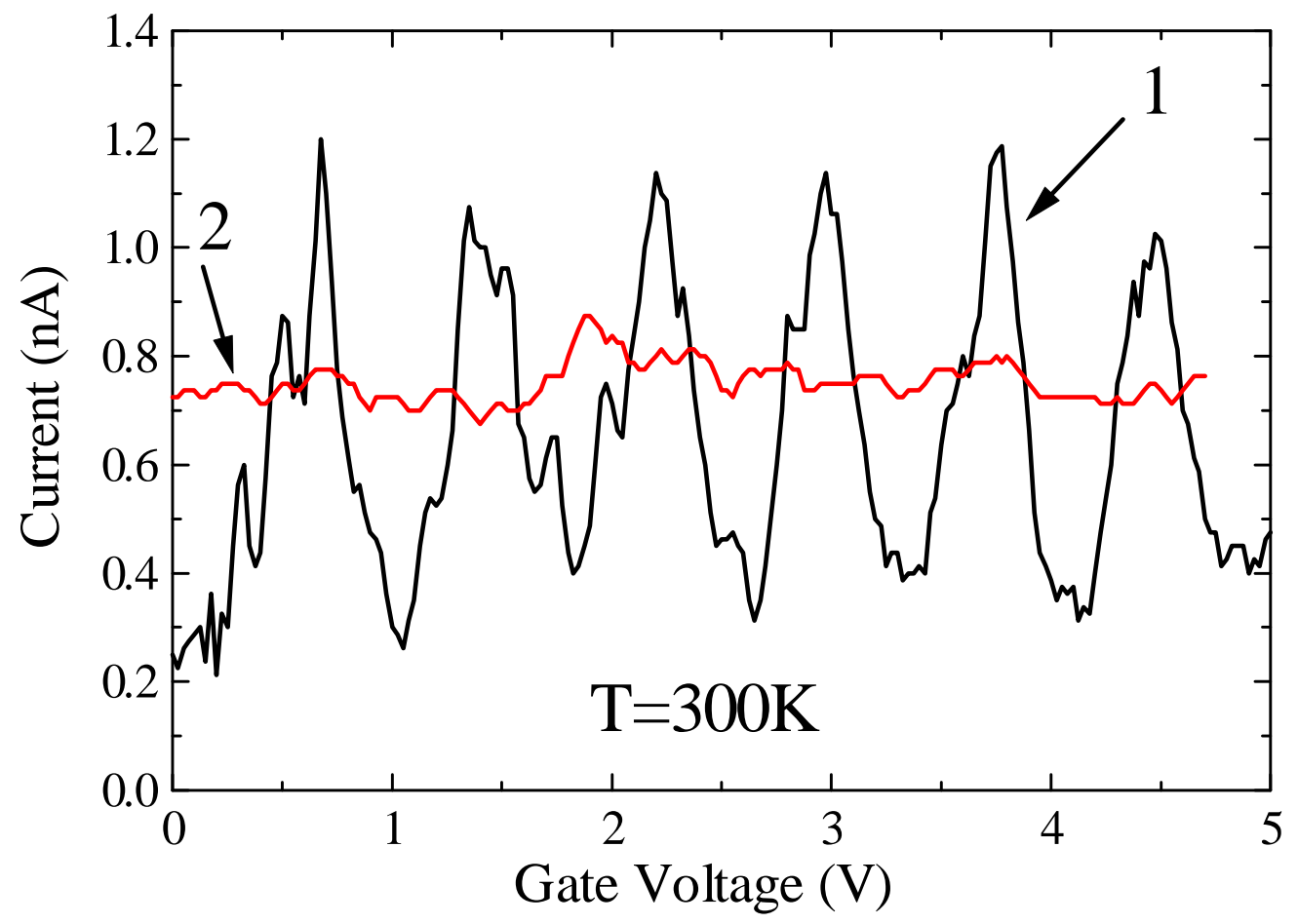




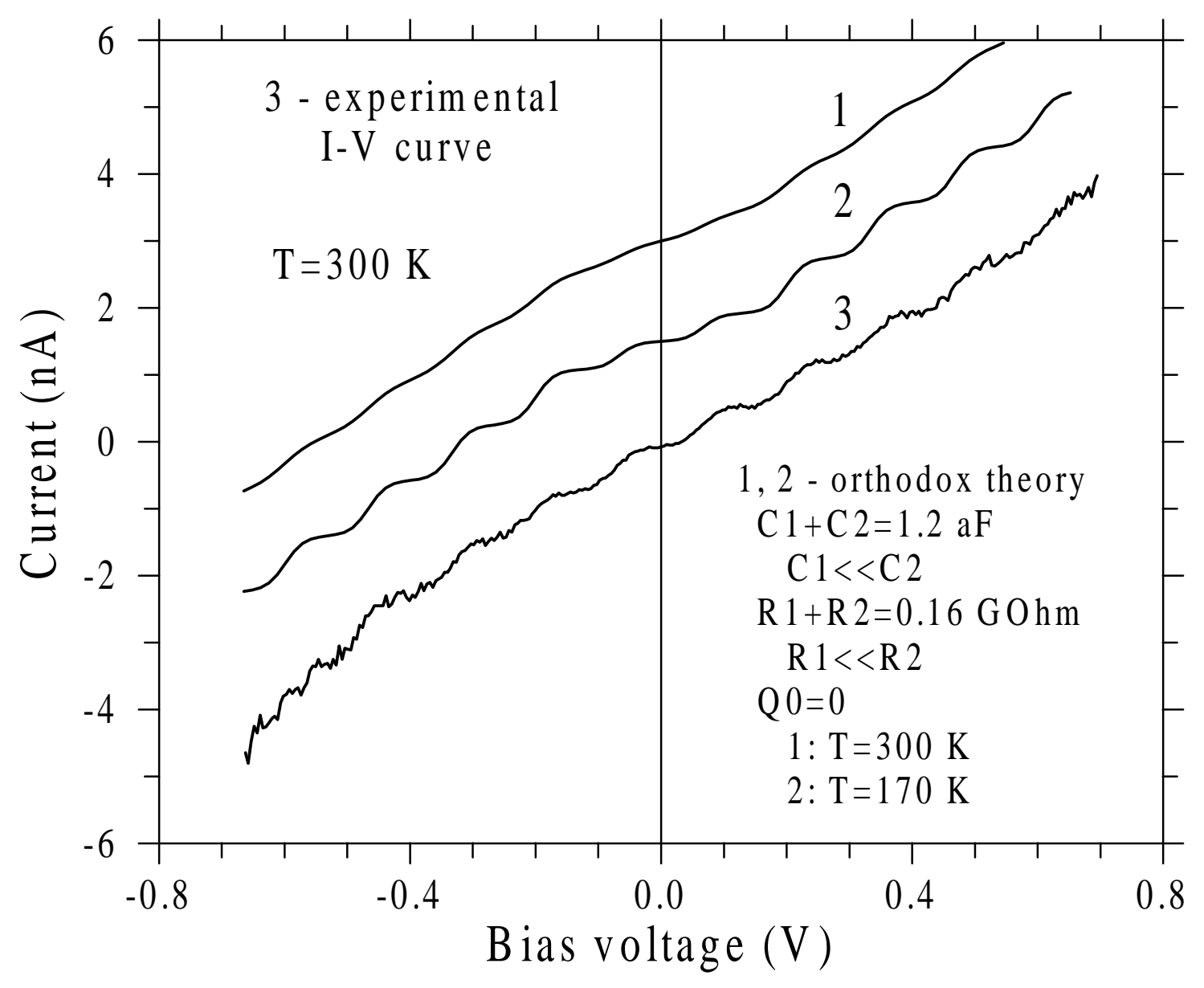

